Int. J. Contemp. Math. Sciences, Vol. 9, 2014, no. 1, 11 - 17

HIKARI Ltd, www.m-hikari.com

http://dx.doi.org/10.12988/ijcms.2014.310114

\title{
Decomposition of Complete Graphs
}

\section{into Union of Stars}

\author{
Ancykutty Joseph* \\ Pemala Kollamparmpil, Athirampuzha, Kerala, India 686562 \\ ancykuttyjoseph@gmail.com \\ *Corresponding author \\ Jinitha Varughese \\ B.K.College, Amalagiri, Kerala, India \\ jinith@gmail.com
}

Copyright (C) 2014 Ancykutty Joseph and Jinitha Varughese. This is an open access article distributed under the Creative Commons Attribution License, which permits unrestricted use, distribution, and reproduction in any medium, provided the original work is properly cited.

\begin{abstract}
Let $\mathrm{S}_{\mathrm{k}+1}$ denote a star with $\mathrm{k}$ edges. Tarsi and Yamamoto et al. have characterized the $S_{k+1}$-decomposability of $K_{n}$ the complete graph. In this paper we study the edge decomposition of both $\mathrm{K}_{\mathrm{n}}$ and the complete bipartite graph $\mathrm{K}_{\mathrm{m}, \mathrm{n}}$ into copies of the union of two edge disjoint stars $S_{p+1}$ and $S_{q+1}$ where $p \neq q$ and $p, q \geq 2$ and obtain the necessary and sufficient conditions for the $S_{p+1} \cup S_{q+1}$-decomposability of $K_{n}$ and $K_{m, n}$.
\end{abstract}

Keywords: Decomposition, Complete graphs, Star

\section{Introduction}

The graphs considered are finite and undirected. The terminology adopted agrees with that in any standard book on graph theory. [2 ] 
$K_{n}$ denotes the complete graph on $\mathrm{n}$ vertices. It has ${ }^{\mathrm{n}} \mathrm{C}_{2}$ edges. $K_{m, n}$, the complete bipartite graph has $\mathrm{m}+\mathrm{n}$ vertices and $\mathrm{mn}$ edges. A star $S_{k+1}$ with $\mathrm{k}+1$ vertices and k edges is nothing but the complete bipartite graph $K_{l, k}$. The star $\mathrm{S}_{\mathrm{k}+1}$ with its centre at 1 and edges joining 1 and $\mathrm{i}, \mathrm{i}=2,3, \ldots, \mathrm{k}$. is denoted by $\mathrm{S}\{1: 2,3, \ldots, \mathrm{k}\}$. A $p$ subset of $\mathrm{V}$ is a subset of size $\mathrm{p}$. The join $\mathrm{G} v \mathrm{H}$ of two vertex disjoint graphs $\mathrm{G}$ and $H$ is the graph with vertex set $V(G) \cup V(H)$ and edge set $E(G) \cup E(H) \cup\{u v: u$ $\in \mathrm{V}(\mathrm{G})$ and $\mathrm{v} \in \mathrm{V}(\mathrm{H})\}$

Let $G$ be any graph and $F=\left\{G_{1}, G_{2}, \ldots G_{k}\right\}$ be a family of subgraphs of $G$. An F decomposition of $G$ is an edge-disjoint decomposition of $G$ into copies of $G_{i}$ for $\mathrm{i}=1,2, \ldots \mathrm{k}$, provided, no $\mathrm{G}_{\mathrm{i}}$ has isolated vertices. Obviously, $\mathrm{k}_{\mathrm{i}}$ is a positive integer. If each $\mathrm{G}_{\mathrm{i}}$ is isomorphic to a graph $\mathrm{H}$ then $\mathrm{G}$ is $\mathrm{H}$ - decomposable or $\mathrm{G}$ has an $\mathrm{H}$ decomposition, denoted by $\mathrm{H} \mid \mathrm{G}$. Clearly, when $\mathrm{H} \mid \mathrm{G}$, then $\mathrm{e}(\mathrm{H}) \mid \mathrm{e}(\mathrm{G})$.If $\mathrm{G}$ is $\mathrm{F}$ decomposable, it necessarily satisfies the condition $\sum_{i=1}^{k} k_{\mathrm{i}} \mathrm{e}\left(\mathrm{H}_{\mathrm{i}}\right)=\mathrm{e}(\mathrm{G})$. For a family F of stars, Lin and Shyu [ 1 ] have given a characterization of the Fdecomposability of $\mathrm{K}_{\mathrm{n}}$.

Tarsi and Yamamoto et al.[4,6] have established the following result." Let $\mathrm{k}$ and $\mathrm{n}$ be positive integers. There exists a $\mathrm{S}_{\mathrm{k}+1}$ decomposition of $K_{, n}$ if and only if $2 \mathrm{k} \leq$ $\mathrm{n}$ and $\mathrm{n}(\mathrm{n}-1) \equiv 0(\bmod 2 \mathrm{k}) . "$

We concentrate on $\mathrm{H}$ - decompositions of $K_{m, n}$, the complete bipartite graph and $K_{, n}$ the complete graph where, $\mathrm{H}=\mathrm{S}_{\mathrm{p}+1} \cup \mathrm{S}_{\mathrm{q}+1}$, the union of two vertex disjoint stars.

We assume that $\mathrm{p} \neq \mathrm{q}$, for otherwise it reduces to star -decomposition. Even then, if either $\mathrm{p}$ or $\mathrm{q}=1$, the problem reduces to decomposition of complete graphs into paths and stars.[5] Hence $p>2$ and q $>3$.Regi T.[ 3] gives the conditions for the $\mathrm{S}_{2} \cup \mathrm{S}_{3}$ decomposability of $K_{n}$ and $K_{m, n}$.

In this paper the $S_{p+1} \cup S_{q+1}, p \geq 2, q \geq 2$, decomposability of complete graphs is characterized. The main results are given in (2.3) and (3.4), theorems A and B.

\section{Decomposition of $K_{m, n}$, the complete bipartite graph into $S_{p+1} \cup$}

$$
\mathbf{S}_{\mathbf{q}+\mathbf{1}}, \mathbf{p} \neq \mathbf{q}
$$

The complete bipartite graph $K_{m, n}$ has $\mathrm{m}+\mathrm{n}$ vertices and $\mathrm{mn}$ edges while $\mathrm{S}_{\mathrm{p}+1}$ $\cup S_{q+1}$ has $p+q+2$ vertices and $p+q$ edges. The stars $S_{p+1}$ and $S_{q+1}$ are vertex disjoint and $K_{m, n}$ is connected. If $K_{m, n}$ is $\mathrm{S}_{\mathrm{p}+1} \cup \mathrm{S}_{\mathrm{q}+1}$-decomposable, then $K_{m, n}$ will be the edge disjoint union of at least two copies of $S_{p+1} \cup S_{q+1}$. For any value of $p$ and $q$, 
there exists the minimal $\mathrm{S}_{\mathrm{p}+1} \cup \mathrm{S}_{\mathrm{q}+1}$ decomposable $K_{m, n}$ with the value two for either $m$ or $n$. Hence, we fix $m, n \geq 2$. The same condition is chosen in [3] also. There are $\mathrm{S}_{\mathrm{p}+1} \cup \mathrm{S}_{\mathrm{q}+1}$-non-decomposable complete bipartite graphs for $\mathrm{m}$ or $\mathrm{n}<.2$.

2.1Example: Let $\mathrm{m}=1, \mathrm{n}=5, \mathrm{p}=2, \mathrm{q}=3 . K_{1,5}$ is not $\mathrm{S}_{3} \cup \mathrm{S}_{4}$ - decomposable , where as

$K_{2,5}$ is decomposable.

The main result of this section is given below.

\subsection{Theorem A:}

Let $\mathrm{m}, \mathrm{n}, \mathrm{p}, \mathrm{q}$ be positive integers such that $\mathrm{m}, \mathrm{n} \geq 2$ and $\mathrm{p} \neq \mathrm{q}$. The necessary and sufficient condition that the complete bipartite graph $K_{m, n}$ is $\mathrm{S}_{\mathrm{p}+1} \cup_{\mathrm{q}+1}$ decomposable is (i) $\mathrm{m}+\mathrm{n} \geq \mathrm{p}+\mathrm{q}+2$ and (ii) $\mathrm{mn} \equiv 0(\bmod (\mathrm{p}+\mathrm{q}))$

Proof: Necessity The conditions (i) and (ii) result immediately from the

$\mathrm{S}_{\mathrm{p}+1} \cup \mathrm{S}_{\mathrm{q}+1}$-decomposability of $K_{m, n}$

Sufficiency: When $\mathrm{mn} \equiv 0(\bmod (\mathrm{p}+\mathrm{q}))$, either $\mathrm{m} \equiv 0(\bmod (\mathrm{p}+\mathrm{q})) \quad$ or $\mathrm{n} \equiv 0(\bmod (\mathrm{p}+\mathrm{q})) . \quad$ It is sufficient to consider any one of them.

$\mathrm{m} \equiv 0(\bmod (\mathrm{p}+\mathrm{q}))$ implies, $\mathrm{m}=\mathrm{k}(\mathrm{p}+\mathrm{q}), \mathrm{k}$ a positive integer and $\mathrm{k} \geq 2$.

Let $V_{1}$ and $V_{2}$ be the subsets into which $V\left(K_{m, n}\right)$ is bipartitioned. Let $\left|V_{1}\right|=m$

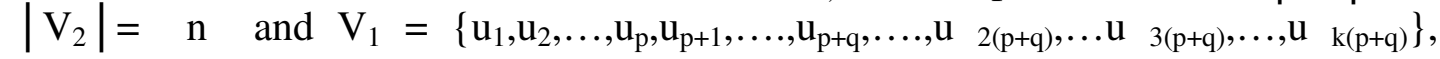
$\mathrm{V}_{2}=\left\{\mathrm{v}_{1}, \mathrm{v}_{2}, \ldots, \mathrm{v}_{\mathrm{n}}\right\}$

$\mathrm{V}_{1}$ is again partitioned into $\mathrm{k}$ subsets of $\mathrm{p}$ vertices and $\mathrm{k}$ subsets of $\mathrm{q}$ vertices.

Case I: $n$ is even. $n=2 r$, for some integer $r \geq 1$.

Partition $\mathrm{V}_{2}$ into $\mathrm{r}=\frac{n}{2}$ subsets of size 2 each.

A p-subset of $\mathrm{V}_{1}$, a q=subset of $\mathrm{V}_{1}$ and a 2-subset of $\mathrm{V}_{2}$ span two disjoint copies of $\mathrm{S}_{\mathrm{p}+1} \cup \mathrm{S}_{\mathrm{q}+1}$.

When all of the p,q,2-subsets are considered, they span a $K_{m, n}$ which is decomposed into $2 \times k \times r=$ nk copies of $S_{\mathrm{p}+1} \cup S_{\mathrm{q}+1}$.

Case II $: n$ is odd. $n=2 r+3 . r \geq 0$. 
Partition $\mathrm{V}_{2}$ into $\frac{n-3}{2}$ 2-subsets and a 3-subset. As in case I,the 2- subsets span 2rk copies of $\mathrm{S}_{\mathrm{p}+1} \cup \mathrm{S}_{\mathrm{q}+1}$.From the 3 -subset, two vertices are chosen in three ways, each pair spanning k copies of $\mathrm{S}_{\mathrm{p}+1} \cup \mathrm{S}_{\mathrm{q}+1} . K_{m, n}$ is decomposed into $2 \mathrm{rk}+3 \mathrm{k}=\mathrm{nk}$ copies of $S_{p+1} \cup S_{q+1}$

This completes the proof.

\subsection{Note:}

The conditions $m, n \geq 2$ and $m+n \geq p+q+2$ guarantee the existence of a $S_{p+1} \cup S_{q+1}$ decomposable $K_{m, n}$

\section{Decomposition of $K_{n}$, the complete graph into $S_{p+1} \cup S_{q+1}, p \neq q$}

The proof methodology adopted here depends on the principle of mathematical induction. Given any $\mathrm{p}$ and $\mathrm{q}$ we have to establish the relation between $\mathrm{p}, \mathrm{q}$ and $\mathrm{n}$ to check whether $K_{n}$ is $\mathrm{S}_{\mathrm{p}+1} \cup \mathrm{S}_{\mathrm{q}+1}$ - decomposable. The proof for the initial values of $\mathrm{p}$ and $\mathrm{q}(2$ and 3$)$ is given separately, even though it is a part of the main theorem. Theorem $\mathrm{A}$ is used in proving Theorem $\mathrm{B}$, the main result here.

3.1 Lemma: If $H \mid G_{1}$ and $H \mid G_{2}$ then $H \mid G_{1} \cup G_{2}$.

3.2 Theorem [3]: $K_{n}$ is $K_{1,2} \cup K_{2}$-decomposable if and only if $\mathrm{n}>4$ and $\mathrm{n} \equiv 0,1(\bmod 3)$

\subsection{Theorem:}

The complete graph $K_{n}$ is $\mathrm{S}_{3} \cup \mathrm{S}_{4}$-decomposable if and only if $\mathrm{n} \geq 10$ and

$$
\mathrm{n} \equiv 0,1(\bmod 5) \text {. }
$$

Proof: Suppose $K_{n}$ is $\mathrm{S}_{3} \mathrm{US}_{4}$-decomposable

Then $5 \mid \mathrm{n} \mathrm{C}_{2}$, implies either $5 \mid \mathrm{n}$ or $5 \mid \mathrm{n}-1$. It follows that $\mathrm{n} \equiv 0,1(\bmod 5)$.

which is equivalent to $\mathrm{n} \equiv 0,1(\bmod 10)$, since 2 and 5 are relatively prime. Also,

$\mathrm{n}>7$, for $\mathrm{S}_{3} \cup \mathrm{S}_{4}$ has seven vertices. $\mathrm{n} \equiv 0,1(\bmod 5)$ implies that $\mathrm{n} \geq 10$.

It is noted that both $K_{5}$ and $K_{6}$ are not $\mathrm{S}_{3} \cup \mathrm{S}_{4}$-decomposable .

Sufficiency: Let $\mathrm{K}_{\mathrm{n}}$ be a complete graph on $\mathrm{n} \geq 10$ vertices and $\mathrm{n} \equiv 0,1(\bmod 5)$. Then, $\mathrm{n}=5 \mathrm{k}$ or $\mathrm{n}=5 \mathrm{k}+1$ for some positive integer $\mathrm{k} \geq 2$. 
Case 1: $\mathrm{n}=5 \mathrm{k}$. for the least value of $\mathrm{n}$, we have $K_{10}$, which is $\mathrm{S}_{3} \cup \mathrm{S}_{4}$-decomposable, illustrated below.

Let $\mathrm{V}\left(K_{10}\right)=\{1,2, \ldots, 10\}$. The nine copies of $\mathrm{S}_{3} \cup \mathrm{S}_{4}$ are,

$\mathrm{S}\{8: 9,10\} \cup S\{4: 5,6,7\}, \mathrm{S}\{9: 1,10\} \cup S\{8: 2,4,5\}, S\{9: 4,5\} \cup S\{10: 2,3,7\}$,

$S\{2: 7,9\} \cup S\{10: 1.4 .5\}, S\{7: 8.9\} \cup S\{3: 4,5,6\}, S\{6: 9,10\} \cup S\{1: 5,7,8\}$,

$S\{6: 1,2\} \cup S\{3: 7,8,9\}, S\{5: 6,7\} \cup S\{1: 2,3,4\}, S\{6: 7,8\} \cup S\{2: 3,4,5\}$

When $\mathrm{k}=3, \mathrm{~K}_{15}$ is $\mathrm{S}_{3} \cup \mathrm{S}_{4}$-decomposable, giving 21 copies of $\mathrm{S}_{3} \cup \mathrm{S}_{4}$,

$S\{1: 13,14,15\} \cup S\{10: 2,11\}, S\{13: 4,5\} \cup S\{2: 14,15\}, S\{1: 10,11,12\} \cup S\{2: 5,6\}$,

$S\{1: 6,7,8\} \cup S\{2: 9,10\}, S\{2: 7,8,11\} \cup S\{3: 4,5\}, S\{3: 6,7,8\} \cup S\{2: 12,13\}$,

$S\{3: 10,11,12\} \cup S\{1: 2,9\}, S\{4: 13,14,15\} \cup S\{3: 9,13\}, S\{4: 5,6,7\} \cup S\{3: 5,14\}$,

$S\{4 ; 8,910\} \cup S\{5,6,7\}, S\{12: 11,13,14\} \cup S\{58,9\}, S\{5: 10,11,12\} \cup S\{13: 14,15\}$

$S\{5: 13,14,15\} \cup S\{6 ; 7,8\}, \quad S\{6 ; 9,10,11\} \cup S\{7: 14,15\}, \quad S\{7: 8,910\} \cup S\{6: 12,13\}$, $S\{7: 11,12,13\} \cup S\{6 ; 14,15\}, S\{8: 13,14,15\} \cup S\{9: 10,11\}, S\{11: 13,14,15\} \cup S\{9: 8,12\}$,

$\mathrm{S}\{8 ; 10,11,12\} \cup \mathrm{S}\{9: 13,14\}, \mathrm{S}\{10 ; 13,14,15\} \cup S\{4: 11,12\}$ and

$S\{15: 9,12,14\} \cup S\{2: 3,4\}$.

For $\mathrm{k}>3$, we have, $\mathrm{E}\left(\mathrm{K}_{5 \mathrm{k}}\right)=\mathrm{E}\left(\mathrm{K}_{5(\mathrm{k}-2)}\right) \cup \mathrm{E}\left(\mathrm{K}_{10}\right) \cup \mathrm{E}\left(\mathrm{K}_{5(\mathrm{k}-2), 10}\right) \ldots \ldots \ldots$ (*) $\left.^{*}\right)$

When $\mathrm{k}=4$, equation(*)yields the edge set of $K_{20}$ as the disjoint union of the edge sets of two $K_{10}$ s and a $K_{10,10}$.each of which is -decomposable by 2.1 (TheoremA ) and the $\mathrm{S}_{3} \cup \mathrm{S}_{4}$-decomposition of $\mathrm{K}_{10}$

By lemma 3.1, $K_{5 k}$ is $\mathrm{S}_{3} \cup \mathrm{S}_{4}$-decomposable for $\mathrm{k}>3$.

Case2: $\mathrm{n}=5 \mathrm{k}+1, \mathrm{k} \geq 2$.

We adopt the same inductive procedure as in case1. When $\mathrm{k}=2$ and k=3 we get $K_{11}$ and $K_{16}$, both $\mathrm{S}_{3} \cup \mathrm{S}_{4}$-decomposable. The relation corresponding to (*) is

$\mathrm{E}\left(K_{5 k+1}\right)=\mathrm{E}\left(K_{5(k-2)}\right) \cup \mathrm{E}\left(\mathrm{K}_{11}\right) \cup \mathrm{E}\left(K_{5(k-2), 11}\right) \ldots \ldots \ldots(* *)$

By lemma 3.1, $K_{5 k+1}$ is $\mathrm{S}_{3} \cup \mathrm{S}_{4}$-decomposable.

The proof is completed. 


\subsection{Theorem B}

The necessary and sufficient condition that the complete graph $K_{n}$ is $\mathrm{S}_{\mathrm{p}+1} \cup \mathrm{S}_{\mathrm{q}+1}$ decomposable, $\mathrm{p} \neq \mathrm{q}$ is

$\mathrm{n}>\mathrm{p}+\mathrm{q}+2$ and (ii) $\mathrm{n} \equiv 0,1(\bmod 2(\mathrm{p}+\mathrm{q}))$.

Proof : (necessity) If $K_{n}$ is $\mathrm{S}_{\mathrm{p}+1} \cup \mathrm{S}_{\mathrm{q}+1}$-decomposable, then $\mathrm{S}_{\mathrm{p}+1} \cup \mathrm{S}_{\mathrm{q}+1}$ is a proper subgraph of $K_{n} \quad$ Hence, $\mathrm{n}>\mathrm{p}+\mathrm{q}+2$.

$\mathrm{e}\left(\mathrm{S}_{\mathrm{p}+1} \cup \mathrm{S}_{\mathrm{q}+1}\right) \mid \mathrm{e}\left(\mathrm{K}_{\mathrm{n}}\right)$ implies $(\mathrm{p}+\mathrm{q}) \mid \mathrm{n} \mathrm{C}_{2}$, equivalently $\mathrm{n} \equiv 0,1(\bmod 2(\mathrm{p}+\mathrm{q}))$.

(sufficiency)The proof is by induction on $\mathrm{n}$. The existence of such a $K_{n}$ is proved for $\mathrm{p}=2$ and $\mathrm{q}=3$ (Theorem 3.3 )

Let $\mathrm{p}>2$ and $\mathrm{q}>3$ and satisfying (i) and (ii) and the graph $K_{m}$ is $\mathrm{S}_{\mathrm{p}+1} \cup \mathrm{S}_{\mathrm{q}+1}$ decomposable

Let $\mathrm{m} \equiv 0,(\bmod 2(\mathrm{p}+\mathrm{q}))$.

The next higher value of $\mathrm{n}$ is $\mathrm{m}+2(\mathrm{p}+\mathrm{q}) \equiv 0(\bmod 2(\mathrm{p}+\mathrm{q}))$ by the induction hypothesis.

$\mathrm{E}\left(K_{m+2(p+q)}\right)=\mathrm{E}\left(K_{m-4}\right) \cup \mathrm{E}\left(\mathrm{K}_{2(\mathrm{p}+\mathrm{q})}\right) \cup \mathrm{E}\left(\mathrm{K}_{\mathrm{m}, 2(\mathrm{p}+\mathrm{q})}\right)$

The graphs on the r.h.s.are $\mathrm{S}_{\mathrm{p}+1} \cup \mathrm{S}_{\mathrm{q}+1}$-decomposable, by the induction hypothesis.

By Lemma 3.1, $K_{m+2(p+q)}$ is $\mathrm{S}_{\mathrm{p}+1} \cup_{\mathrm{q}+1}$-decomposable, When $\mathrm{m} \equiv 0(\bmod 2(\mathrm{p}+\mathrm{q}))$, $\mathrm{m}+1 \equiv 1(\bmod 2(\mathrm{p}+\mathrm{q}))$.

From, $K_{m+l}=K_{m} v K_{l}$ we have, $\mathrm{E}\left(K_{m+1}\right)=\mathrm{E}\left(K_{m}\right) \cup \mathrm{E}\left(K_{l, m}\right)$.

By the induction hypothesis, Theorem $\mathrm{A}$ and lemma 3.1, $K_{m+1}$ is $\mathrm{S}_{\mathrm{p}+1} \mathrm{US}_{\mathrm{q}+1^{-}}$ decomposable.

By induction principle, for any $\mathrm{n}$ satisfying the conditions ((i) $\mathrm{n}>\mathrm{p}+\mathrm{q}+2$ and (ii) $\mathrm{n} \equiv 0,1(\bmod 2(\mathrm{p}+\mathrm{q})) K_{n}$ is $\mathrm{S}_{\mathrm{p}+1} \cup \mathrm{S}_{\mathrm{q}+1}$-decomposable.

Conclusion: We have given, in this paper, a characterization for the $S_{p+1} \cup S_{q+1}$, $\mathrm{p} \geq 2, \mathrm{q} \geq 2$, decomposability of complete graphs. Similar results for other graphs will be given in another paper. 
Acknowledgement. The first author is grateful to the University Grants Commission for the financial support under the scheme of major research project vide letter No.F.No.39-60/2010(SR) dated 13.1.2011

\section{References}

1. Lin.C and T.W-Shyu: A necessary and sufficient condition for the star decomposition of complete graphs, J. Graph Theory 23 (1996) 361-364.

2. Parthasaradhy K.R: Basic Graph Theory, Tata MacGraw Hill.

3. Regi T.: A Study on Decomposition Problems in Graph Theory, Ph.D thesis University of Kerala, 2005.

4. Tarsi M.: On the decomposition of a graph into stars, Discrete Mathematics, 36 (1981) 299-304.

5. T. W-Shyu; Decomposition of complete graphs into paths and stars, Discrete Mathematics 310 (2010 ) 2164-2169.

6. Yamamoto S., Ikeda H., Shige-eda S, Ushio K. and Hamada N.: On claw decomposition of complete graphs and complete bigraphs, Hiroshima Math. J. 5 (1975) 33-42.

Received: October 11, 2013 